\title{
Impact of Covid-19 on Mental Health: A Bibliometric Assessment of Publications from India
}

\author{
Sandeep Grover ${ }^{1,}$, BM Gupta ${ }^{2}$, Ghouse Modin Mamdapur ${ }^{3}$ \\ 'Post Graduate Institute of Medical Education and Research, Chandigarh, INDIA. \\ 2Formerly with CSIR-NISTADS, New Delhi, Delhi, INDIA. \\ ${ }^{3}$ Synthite Industries (P) Ltd, Kolenchery, Kerala, INDA.
}

\section{ABSTRACT}

Aim: The paper assesses the India's research output on "Impact of Covid-19 on Mental Health" indexed in Scopus database. Materials and Methods: The Scopus database was used to search for the articles published from India. Results: The search showed that since the onset of the pandemic upto $24^{\text {th }}$ of April 2021, 1210 publications emerged from India, amounting to $6.87 \%$ share of the global output on the topic and averaged 5.97 citations per paper. India stood at $5^{\text {th }}$ position in terms of number of publications on mental health, with highest number of publications emerging from United States $(26.9 \%)$, followed by United Kingdom (13.35\%), China $(9.83 \%)$ and Italy $(8.27 \%)$. About one-third $(30.91 \%)$ of the publications involved international collaborations, with maximum number of collaborations were with United States, followed by United Kingdom, Australia, Italy, Canada, and Brazil. The most common keywords in the research included, 'mental health', followed by 'anxiety' and 'depression'. The research output came from 478 organizations, with maximum research coming from National Institute of Mental Health and Neuro Sciences (NIMHANS), Bangalore, All India Institute of Medical Sciences (AIIMS), New Delhi and Postgraduate Institute of Medical Education \& Research (PGIMER), Chandigarh. About
\end{abstract}

three-fourth $(76.85 \%)$ of the mental health research emerged from institute other than these 3 institutes. Five out of the 10 most productive authors and 5 out of the most impactful authors were from institutes other than the 3 major institutes. Conclusion: To conclude, this bibliometric analysis suggest that, researchers from India contributed to about 7\% of the global mental health research on Covid-19 and India stood at $5^{\text {th }}$ position among the various countries in terms of global mental health research on Covid-19.

Key words: Covid-19, Mental health, Depression, Anxiety, Bibliometrics, India.

\section{Correspondence}

Prof. Sandeep Grover,

Post Graduate Institute of Medical Education and Research, Chandigarh-160012, INDIA.

Email id: drsandeepg2002@yahoo.com

DOI: 10.5530/jyp.2021.13s.73

\section{INTRODUCTION}

The Covid-19 pandemic, emerging from China and then spreading across the globe, has brought the world to a standstill, with multitudes of negative impact in the form of spread of infection, the requirement of hospitalization, the need for respiratory support, and mortality. Additionally, the pandemic has also led to lockdown, with significant adverse outcomes in the form of an increase in mental health morbidity, loss of jobs, domestic violence, self-harm and suicide, negative impact on sexual life, financial difficulties, crisis for the migrant workers, etc. ${ }^{1-8}$ Further, studies also show that Covid-19 infection also leaves a scar on the mental health of the people who suffer from the illness in the form of increased rates of depression, anxiety, cognitive impairment, and dementia etc. ${ }^{9,10}$ Researchers across the globe have attempted to assess the various mental health outcomes of the Covid-19 pandemic. It is said that the number of publications focusing on the mental health aspect of the ongoing pandemic has risen exponentially with the passing time.

Few bibliometric/scientometric studies related to "Impact of Covid-19 on Mental Health" exist. Maalouf et al. ${ }^{11}$ examined 277 publications related to the "Covid-19 pandemic and mental health" published since the onset of the pandemic up to $26^{\text {th }}$ August 2020 and compared it with the literature output on the West Africa Ebola and H1N1 outbreaks. Despite the shorter time since the beginning of the Covid-19 pandemic, compared to the Ebola and H1N1 outbreaks (127 for Ebola and 327 for $\mathrm{H} 1 \mathrm{~N} 1$ ), the authors found a much greater number of publications on the mental health aspect of Covid-19 $(n=3070)$. Gul et al. ${ }^{12}$ examined the trends in publication on "Covid-19 and mental health" published from the outbreak of the pandemic to up to July 2020, in 195 journals, with a focus on top-cited documents, productive countries, institutions, journals, authorship and collaboration, the most frequent keywords and funding bodies. Dube and Dadhe ${ }^{13}$ explored the global research productivity on mental health research during the Covid-19 pandemic published from $1^{\text {st }}$ January 2020 to $5^{\text {th }}$ November 2020 by identifying key countries, organizations, authors, and journals and found 1690 records on the same. In another study, the authors examined the recent trends in global research publications (15223 papers published up to $21^{\text {st }}$ March 2021) on "Impact of Covid-19 on Mental Health". The authors found 15223 publications emerging from 158 countries, with each publication registering 8.9 citations and $13.77 \%$ publications receiving funding support for the work. In terms of the top 10 most productive countries, authors reported that the maximum number of publications emerged from the USA, followed by the U.K. and China. In terms of the most productive organizations, the maximum number of publications emerged from Harvard Medical School, USA; University of Toronto, Canada; and King's College, London, U.K. The National University of Singapore (51.84 and 5.83), King's College, London, U.K. (27.23 and 3.06), Huazhong University of Science and Technology, China (23.65 and 2.66) were found to be the most impactful organizations in terms of citations per paper and relative citation index. ${ }^{14}$ This study also showed that India stood $5^{\text {th }}$ in terms of publications on research on 
the mental health aspect of Covid-19. However, no bibliometric study has focused on the mental health research output on Covid-19 from India. Accordingly, this study aims to evaluate the research on mental health and Covid-19 from India, focusing on identifying India's global share, international collaborative publications, and citation impact, most influential organizations, authors, and journals, the subject focus of research and analysis of high-cited papers.

\section{MATERIALS AND METHODS}

For identifying, retrieving and downloading global publications data on "Impact of Covid-19 on Mental Health", the data was sourced from the Scopus database (http://www.scopus.com) up to 24.4.2021. A set of keywords related to "Covid-19" and "Mental Health" were used in "Keyword tag" as well as in "Article Title tag" (joined by Bolean operator "or") simultaneously to get global publication data (consisting of 17608 records). The above described search strategy was refined by country of publication (including India) to get the data on country-wise publications. India's publication output comprised of 1210 records. The search strategy was further refined to get statistics on India's output by subject, collaborating country, organization, author and journal. Citations to publications were counted from date of their publication till 14 April 2020.

The various keywords used to identity the publications included, "Covid 19", “2019 novel coronavirus", "coronavirus 2019”, "coronavirus disease 2019", "2019-novel CoV”, "2019 ncov", "Severe acute respiratory syndrome coronavirus 2" or "SARS-CoV-2" , mental health, India, in different combinations for searching the title, keywords and country. The available literature was analysed for the titles, authors, year of publication, affiliations, type of document, fields of interest, funding sources, keywords, citation frequency, etc.

\section{RESULTS}

\section{Publication Growth}

The global and Indian research publications in the field "Impact of Covid-19 on Mental Health" consisted of 17608 and 1210 publications and the citation impact of publications from India averaged to 5.97 citations per paper (CPP). India stood at $5^{\text {th }}$ position in terms of number of publications on mental health, with highest number of publications emerging from USA (26.9\%), followed by U.K. (13.35\%), China (9.83\%) and Italy (8.27\%).

Only a small proportion of the publications $(n=106 ; 8.76 \%)$ from India were based on funding by national and international funding agencies and these publications received 3680 citations, averaging 8.89 CPP. In terms of type of publications, $57.44 \%(n=695)$ papers appeared as articles, $19.34 \%(n=234)$ as letters, $15.29 \%$ $(n=185)$ as reviews, $2.98 \%(n=36)$ as editorials, $2.81 \%(n=34)$ as notes and other type of publications accounted for $<1 \%$ of the total publications from India.

\section{International Collaboration}

The share of international collaborative papers $(n=374)$ with one of the authors from India was $30.91 \%$ of the total outoput on this theme. The international collaborative papers had linkages with authors from 50 countries, with maximum number of collaborations with USA $(n=162 ; 43.32 \%)$, followed by U.K. $(n=110 ; 29.41 \%)$, Australia $(n=63$; $16.84 \%)$, Italy $(n=51 ; 13,64 \%)$, Canada $(n=44 ; 11.76 \%)$, and Brazil $(n=43$; $11.5 \%$ ) (Figure 1).

In terms of South Asian Association for Regional Cooperation (SAARC) Countries, maximum number of collaborations were with Bangladesh $(n=42 ; 11.23 \%)$ and this was followed by collaboration with Nepal

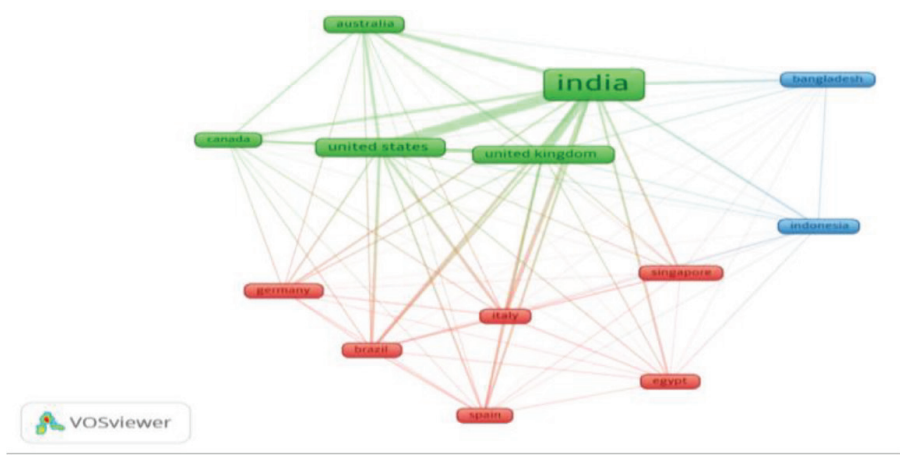

Figure 1: Collaboration of Researchers from India with Researchers from other countries.

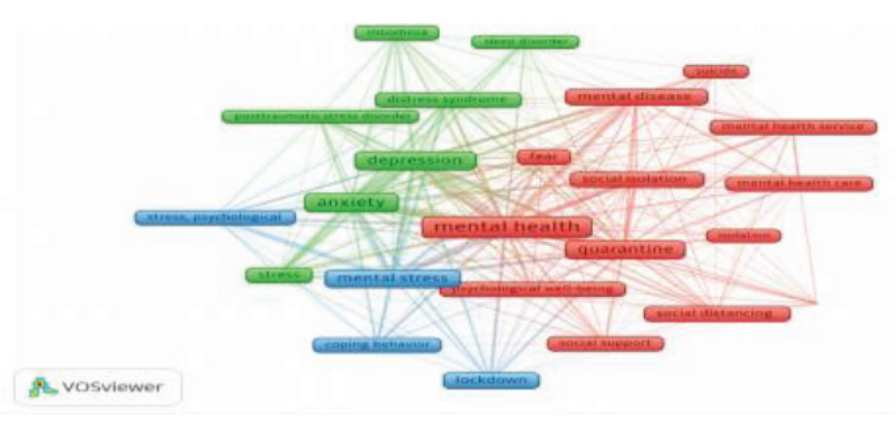

Figure 2: Commonly used keywords in the mental health research on Covid-19 from India.

$(n=27 ; 7.22 \%)$, Pakisthan $(n=24 ; 6.42 \%)$, Sri Lanka $(n=5 ; 1.34 \%)$ and Myanmar $(n=1 ; 0.27 \%)$. Together these papers amounted to 77 publications, which received 935 citations, averaging to $12.14 \mathrm{CPP}$.

Of the 77 international collaborative papers, 16 papers (20.78\%) involved bilateral collaboration (i.e., authors from India and one more country) and 61 papers $(79.22 \%)$ involved multilateral collaboration (partcipitation of authors from more than 2 countries along with India).

\section{Significant Keywords.}

Keyword co-occurrence in research publications offers an alternative approach to identify and highlights the key research trends. A total of 24 keywords were identified from publications emerging from India with respect to the "Impact of Covid-19 on Mental Health". The most common keyword, included, "mental health", followed by "anxiety" and "depression" (Figure 2).

\section{Top 25 Most Productive Organizations from India}

The publication on mental health aspect of Covid-19, came from 478 organizations, with 1-5 publications emerging from 350 organizations, 6-10 papers from 90 organizations, 11-20 papers from 27 organization, 21-50 papers from 8 organizations, and 51-128 papers emerging from 3 organizations. The productivity of top 25 most productive organizations varied from 11 to 128 publications per organization; together authors from these 25 organizations contributed to $63.8 \%(n=772)$ publications share and $65.4 \%(n=4724)$ citations share to India's total publications and citations on the topic. The detailed scientometric profile of top 8 most productive and top 8 most impactful organizations is presented in Table 1. 
Table 1: Top 8 Most Productive and Top 8 Most Impactful Organizations in India's Research on “Impact of Covid-19 on Mental Health”.

\begin{tabular}{|c|c|c|c|c|c|c|c|c|}
\hline S.No & Name of the Organization & TP & $\mathrm{TC}$ & CPP & HI & ICP & $\%$ ICP & $\mathrm{RCl}$ \\
\hline \multicolumn{9}{|c|}{ Top 8 Most Productive Organizations } \\
\hline 1 & National Institute of Mental Health and Neuro Sciences, Bangalore & 128 & 855 & 6.68 & 14 & 27 & 21.09 & 1.12 \\
\hline 2 & All India Institute of Medical Sciences, New Delhi & 84 & 265 & 3.15 & 8 & 22 & 17.19 & 0.53 \\
\hline 3 & Postgraduate Institute of Medical Education \& Research, Chandigarh & 68 & 416 & 6.12 & 11 & 11 & 8.59 & 1.02 \\
\hline 4 & Datta Meghe Institute of Medical Sciences (Deemed to be University) & 48 & 40 & 0.83 & 3 & 5 & 3.91 & 0.14 \\
\hline 5 & Saveetha Institute of Medical and Technical Sciences & 44 & 5 & 0.11 & 1 & 2 & 1.56 & 0.02 \\
\hline 6 & Saveetha Dental College and Hospital & 41 & 4 & 0.10 & 1 & 2 & 1.56 & 0.02 \\
\hline 7 & King George’s Medical University & 35 & 665 & 19.00 & 10 & 14 & 10.94 & 3.18 \\
\hline 8 & Manipal Academy of Higher Education & 34 & 281 & 8.26 & 8 & 16 & 12.50 & 1.38 \\
\hline \multicolumn{9}{|c|}{ Top 8 Most Impactful Organizations } \\
\hline 1 & Tata Memorial Hospital & 12 & 428 & 35.67 & 2 & 4 & 3.13 & 5.97 \\
\hline 2 & Jawaharlal Institute of Postgraduate Medical Education and Research & 33 & 802 & 24.3 & 8 & 6 & 4.69 & 4.07 \\
\hline 3 & King George’s Medical University & 35 & 665 & 19 & 10 & 14 & 10.94 & 3.18 \\
\hline 4 & BKL Walawalkar Rural Medical College, Kasarwadi & 13 & 143 & 11 & 5 & 10 & 7.81 & 1.84 \\
\hline 5 & Jamia Millia Islamia & 17 & 173 & 10.18 & 6 & 5 & 3.91 & 1.7 \\
\hline 6 & Manipal Academy of Higher Education & 34 & 281 & 8.26 & 8 & 16 & 12.5 & 1.38 \\
\hline 7 & Jawaharlal Nehru Medical College, Wardha & 19 & 142 & 7.47 & 2 & 2 & 1.56 & 1.25 \\
\hline 8 & National Institute of Mental Health \& Neuro Sciences, Bangalore & 128 & 855 & 6.68 & 14 & 27 & 21.09 & 1.12 \\
\hline
\end{tabular}

TP=Total Papers; TC=Total Citations; CPP=Citations Per Paper; ICP=International Collaborative Papers; HI=H-Index; RCI=Relative Citation Index

\section{Top 25 Most Productive Authors from India}

975 authors participated in research on impact of Covid-19 on mental health with 910 authors publishing 1-5 paper each, 53 authors published 6-10 papers each, 8 authors publishing 11-20 papers each and 4 authors publishing $>20$ papers (Table 2 ). The research productivity of top 25 most productive authors varied from 6 to 28 publications per author. Together they contributed to about one-fourth $(n=289 ; 23.88 \%)$ share of publications and slightly more than one third $(n=2716 ; 37.6 \%)$ share of citations for total publications and citations (Table 2). Seven of top 25 authors registered their publications output above the group average of 11.56: S. Grover (28 papers), D. Banerjee (26 papers), A. Mehra and S. Sahoo (23 papers each), R. Ransing (20 papers), S.K. Kar (18 papers) and V.Menon (15 papers). Four of the top 25 authors registered their citation per paper and relative citation index above the group average (9.40 and 1.57) of all authors with R.P.Rajkumar (75.44 and 12.64) at the top of the list, followed by S.K. Kar (28.67 and 4.8), S.S. Chatterjee (12.13 and 2.03) and D. Banerjee (12.12 and 2.03).

\section{Medium of Research Communication}

The majority of the publications emerged as journal publications $(n=1191 ; 98.43 \%)$ spread over 479 journals. Of the 479 journals reporting 1191 articles, 447 published 1-5 papers each, 17 journals publishing 6-10 papers each, 8 journals publishing 11-20 papers each and 7 journals publishing 21-123 papers. The top 25 most productive journals accounted for $41.9 \%$ share of total Indian output in journals and $57.47 \%$ citations share of India.

Asian Journal of Psychiatry was the topmost productive journal (with 123 papers), followed by International Journal of Research In Pharmaceutical Sciences (50 papers), Indian Journal of Psychiatry (33 papers), International Journal of Current Research and Review (30 papers), Indian Journal of Forensic Medicine and Toxicology (26 papers), Diabetes and Metabolic Syndrome Clinical Research and Reviews (25 papers), International Journal of Social Psychiatry (21 papers), Frontiers in Psychiatry and Psychiatry
Research (17 papers each). In terms of citation per paper, Brain Behavior and Immunity led in ranking (57.78), followed by Diabetes and Metabolic Syndrome Clinical Research and Reviews (20.96), Psychiatry Research (17.88), Asian Journal of Psychiatry (16.71), PLOS One (9.8), International Journal of Social Psychiatry (6.81), International Journal of Environmental Research \& Public Health (6.5), Dermatologic Therapy (6.0) and Indian Journal of Psychiatry (4.58).

\section{High Cited Papers}

Of the total research output on "Impact of Covid-19 on Mental Health" only 20 (1.65\% share) papers accumulated 51 to 845 CPP (cumulative total 3212 citations) since their publication, averaging to 160.6 CPP. The distribution of these 20 high-cited papers was skewed. Eleven papers accumulated citations in the range 51-95 per paper, 5 papers were cited $134-188$ times and 4 papers were cited 308-845 times. Of the 20 high-cited papers, 9 were published as articles, 5 as letters, 4 as review papers and 2 as editorials. Of the 20 highly cited papers, 6 came from authors of single organizations (non-collaborative papers) and 14 had authors from two or more organizations (5 national collaborative and 9 international collaborative papers). These 20 highcited papers appeared across different journals, of which 5 papers were published in Asian Journal of Psychiatry, 2 papers each in Diabetes and Metabolic Syndrome Clinical Research and Reviews, Brain Behavior and Immunity and Psychaitry Research and 1 paper each in 9 other journals.

\section{DISCUSSION}

Covid-19 pandemic has emerged as a significant public health issue in the latter half of 2019 and is still making havoc in many countries worldwide. It is suggested that, although everyone is not affected by the virus, the pandemic has had a significant negative impact on the mental health of everyone. ${ }^{1}$ In this hour of crisis, it is important to understand the emerging mental health issues in people, especially in the crosscultural background. Hence, it is important to share the knowledge in 
Table 2: Top 10 Most Productive and Most Impactful Indian Authors in Research on “Impact of Covid-19 on Mental Health”.

\begin{tabular}{|c|c|c|c|c|c|c|c|c|c|}
\hline S.No & Name of the Author & Affiliation of the Author & TP & TC & CPP & $\mathrm{HI}$ & ICP & $\% \mathrm{ICP}$ & $\mathrm{RCl}$ \\
\hline \multicolumn{10}{|c|}{ Top 10 Most Productive Authors } \\
\hline 1 & S. Grover & Postgraduate Institute of Medical Education \& Research, Chandigarh & 28 & 177 & 6.32 & 8 & 2 & 7.14 & 1.06 \\
\hline 2 & D. Banerjee & National Institute of Mental Health and Neuro Sciences, Bangalore & 26 & 315 & 12.12 & 7 & 5 & 19.23 & 2.03 \\
\hline 3 & A. Mehra & Postgraduate Institute of Medical Education \& Research, Chandigarh & 23 & 173 & 7.52 & 8 & 2 & 8.70 & 1.26 \\
\hline 4 & S. Sahoo & Postgraduate Institute of Medical Education \& Research, Chandigarh & 23 & 173 & 7.52 & 8 & 2 & 8.70 & 1.26 \\
\hline 5 & R. Ransing & BKL Walawalkar Rural Medical College, Kasarwadi & 20 & 166 & 8.30 & 7 & 16 & 80.00 & 1.39 \\
\hline 6 & S.K.Kar & King George’s Medical University, Lucknow & 18 & 516 & 28.67 & 8 & 12 & 66.67 & 4.80 \\
\hline 7 & V. Menon & $\begin{array}{l}\text { Jawaharlal Institute of Postgraduate Medical Education and Research, } \\
\text { Puducheery }\end{array}$ & 15 & 113 & 7.53 & 6 & 6 & 40.00 & 1.26 \\
\hline 8 & S. Shoib & Jawahar Lal Nehru Memorial Hospital,Srinagar & 11 & 27 & 2.45 & 3 & 8 & 72.73 & 0.41 \\
\hline 9 & N. Manjunatha & National Institute of Mental Health and Neuro Sciences, Bangalore & 9 & 45 & 5.00 & 4 & 0 & 0.00 & 0.84 \\
\hline 10 & R.P. Rajkumar & $\begin{array}{l}\text { Jawaharlal Institute of Postgraduate Medical Education and Research, } \\
\text { Puducheery }\end{array}$ & 9 & 679 & 75.44 & 3 & 0 & 0.00 & 12.64 \\
\hline \multicolumn{10}{|c|}{ Top 10 Most Impactful Authors } \\
\hline 1 & R.P. Rajkumar & $\begin{array}{l}\text { Jawaharlal Institute of Postgraduate Medical Education and Research, } \\
\text { Puducheery }\end{array}$ & 9 & 679 & 75.44 & 3 & 0 & 0.00 & 12.64 \\
\hline 2 & S.K. Kar & King George’s Medical University, Lucknow & 18 & 516 & 28.67 & 8 & 12 & 66.67 & 4.80 \\
\hline 3 & S.S. Chatterjee & Diamond Harbour Medical College \& Hospital, Kolkata & 8 & 97 & 12.13 & 4 & 5 & 62.50 & 2.03 \\
\hline 4 & D. Banerjee & National Institute of Mental Health and Neuro Sciences, Bangalore & 26 & 315 & 12.12 & 7 & 5 & 19.23 & 2.03 \\
\hline 5 & R. Ransing & BKL Walawalkar Rural Medical College, Kasarwadi & 20 & 166 & 8.30 & 7 & 16 & 80.00 & 1.39 \\
\hline 6 & S. Sarkar & All India Institute of Medical Sciences, New Delhi & 7 & 54 & 7.71 & 3 & 0 & 0.00 & 1.29 \\
\hline 7 & V. Menon & $\begin{array}{l}\text { Jawaharlal Institute of Postgraduate Medical Education and Research, } \\
\text { Puducheery }\end{array}$ & 15 & 113 & 7.53 & 6 & 6 & 40.00 & 1.26 \\
\hline 8 & A. Mehra & Postgraduate Institute of Medical Education \& Research, Chandigarh & 23 & 173 & 7.52 & 8 & 2 & 8.70 & 1.26 \\
\hline 9. & S. Sahoo & Postgraduate Institute of Medical Education \& Research, Chandigarh & 23 & 173 & 7.52 & 8 & 2 & 8.70 & 1.26 \\
\hline 10 & S. Grover & Postgraduate Institute of Medical Education \& Research, Chandigarh & 28 & 177 & 6.32 & 8 & 2 & 7.14 & 1.06 \\
\hline
\end{tabular}

TP=Total Papers; TC=Total Citations; CPP=Citations Per Paper; ICP=International Collaborative Papers; HI=H-Index; RCI=Relative Citation Index

the form of published research, which others can assess to provide better health care services to the people in this hour of crisis.

This paper analyzes the research output (1210 papers) from India on the "Impact of Covid-19 on Mental Health" based on few bibliometric indicators based on publications covered in the Scopus database. Our findings suggest that researchers from India contributed $6.87 \%$ share to global research output (17608 papers) on the topic, with an average citation impact of $5.97 \mathrm{CPP}$. In terms of the total number of publications, India stood at the fifth position in the world research output on the mental health aspect of Covid-19. What do these figures suggest?. In general, it has been shown that there is always a big gap in terms of the proportion of mental health research, which emerges from developed countries and that from developing countries. A study, which evaluated the published mental health research by using ISI Web of Science database (19922001), showed that only $6 \%$ of the mental health research emerges from low and middle-income countries, with 14 out of the 54 high-income countries accounting for $90 \%$ of the mental health research. ${ }^{15}$ Two of the recent bibliometric studies evaluating the research output on schizophrenia and bipolar disorder from India suggest that only $2 \%$ of the world mental health research on these disorders emerges from India, and India is at the 13th position on the contribution of research on schizophrenia and bipolar disorder. ${ }^{16,17}$ Suppose one compares the research output on mental health aspects of Covid-19, emerging from India. In that case, it can be said that the percentage contribution of research on Covid-19 from India has been higher than that has been estimated earlier. This can be understood as a healthy sign for mental health research from India. Many factors, such as lower rejection rates and research focusing on contemporary topics, could have contributed to this higher proportion of research emerging from India. In terms of international collaborations, 30.9\% of the papers with one of the authors from India involved international collaboration, with a maximum percentage of collaborations with researchers from the USA, followed by the U.K., Australia, Italy, Canada, and Brazil. Further, there were also collaborations with other SAARC countries, with many collaborations with Bangladesh and Nepal. These findings suggest that the proportion of collaborative papers on the mental health aspect of Covid-19 has been more than that reported for Schizophrenia and Bipolar disorder. ${ }^{16,17}$ These findings again suggest that Covid-19 gave more opportunities to the researchers to collaborate more freely. This could be attributed to the fact that most of the publications on mental health aspects of Covid-19 emerged as viewpoints, review articles, letters to the editors, online surveys, etc. Many of these possibly did not involve going through the rigorous process of ethical clearance and data collection over a long period. Hence, these collaborations were perhaps more successful. The other reason for the higher rate of collaboration could be a genuine increase in the collaborations in online webinars, which have provided additional opportunities to collaborate. The most common keywords which were identified were mental health, followed by anxiety and depression. These keywords reflect the mental impact of the Covid-19 pandemic. In general, available studies suggest 
an increase in the prevalence of common mental disorders among the general population. ${ }^{18}$ Another important finding of the present study is the contribution of researchers from institutes other than NIMHANS, Bangaluru; AIIMS, New Delhi; and PGIMER, Chandigarh to the mental health research on Covid-19. About three-fourth (76.85\%) of the mental health research emerged from institutes other than these three institutes. This proportion is higher than the research out from institutes other than these three institutes on schizophrenia and bipolar disorder. ${ }^{16,17}$

Another thing that is evident from the data analysis is that many institutes contributed to the research on Covid-19 and mental health. However, an essential aspect is that the papers emerging from institutes (other than the three major institutes) had more impact on citations. This was also reflected in most productive authors, with 5 out of the ten most productive authors and 5 out of the most impactful authors were from institutes other than the three major institutes. In terms of the most impactful authors, again, the authors from institutes other than the three major institutes had more impact on the number of citations and CPP. These findings suggest that Covid-19 led to broader distribution of research from India, with researchers from institutes other than the two major institutes contributing to a larger share compared to the research on other mental disorders, and the researchers from these institutes had more impact in terms of the number of citations and CPP.

The majority of the papers on Covid-19 and mental health were published in journals, with a maximum number of publications in the Asian Journal of Psychiatry (123 articles), followed by the International Journal of Research In Pharmaceutical Sciences (50 papers) and Indian Journal of Psychiatry (33 documents). Publications of the maximum number of papers in the Asian Journal of Psychiatry could be due to the author-friendly editorial policies of faster review of the articles, and publication of the manuscripts as a letter to editors, as has been reported by the Editor's themselves..$^{19}$ This possibly reduced the rejection rates and gave more opportunity and encouragement to the authors to publish their research. These policies are worth considering in the future too, for mental health research in general, to reduce the 10/90 gap. Additionally, papers published in the Asian Journal of Psychiatry received the highest number of citations, suggesting that the quality of the papers was not compromised with the expedition of the time to publish. Limitations of the present study must be kept in mind while interpreting the findings of the present study. No attempt was made to evaluate the type and content of the research in terms of original research papers, case reports/case series, review articles. Hence, the increase in the research output may not be a reflection of the publication of original research articles. Similarly, the citation count was also limited to Scopus search Engine, which usually reflects a lower citation count compared to the google scholar. This could have led to a lower estimation of the citations. However, a major strength of this analysis is use of Scopus search Engine, which has more broader coverage than the Pubmed. It covers all the journals listed in the Pubmed and also covers journals not listed in the Pubmed.

To conclude, this bibliographic analysis suggests that researchers from India contributed to about $7 \%$ of the global mental health research on Covid-19. India stood at $5^{\text {th }}$ position among the various countries in terms of international mental health research. The present analysis also suggests that a significant proportion of mental health research on Covid-19 emerged from institutes other than NIMHANS-Bangalore, AIIMSNew Delhi, and PGIMER-Chandigarh. Further, nearly half of the top 10 most productive researchers and most impactful researchers in terms of number of citations and CPP came from institutes other than NIMHANS-Bangalore, AIIMS-New Delhi, and PGIMER- Chandigarh. The maximum number of documents were published in the Asian Journal of Psychiatry. These findings suggest that the Covid-19 pandemic has led to a change in the contribution of mental health research from researchers from India, with a significant increase in India's research contribution. Further, these findings suggest that the research base in India is becoming more wide-base, with research on mental health spread across many more institutes than earlier.

\section{CONFLICT OF INTEREST}

The authors declare no conflict of interest.

\section{ABBREVIATIONS}

Covid-19: 2019 Novel Corona Virus disease.

\section{REFERENCES}

1. Grover S, Dua D, Sahoo S, Mehra A, Nehra R, Chakrabarti S. Why all Covid-19 hospitals should have mental health professionals: the importance of mental health in a worldwide crisis! Asian J Psychiatr. 2020 Jun;51:102147. doi: 10.1016/j.ajp.2020.102147.

2. Grover S, Mehra A, Sahoo S, Avasthi A, Tripathi A, D'Souza A, et al. State of mental health services in various training centers in India during the lockdown and Covid-19 pandemic. Indian J Psychiatry. 2020 Jul-Aug:62(4):363-9. doi: 10.4103/psychiatry.IndianJPsychiatry_567_20, PMID 33165355.

3. Grover S, Mehra A, Sahoo S, Avasthi A, Tripathi A, D'Souza A, et al. Impact of Covid-19 pandemic and lockdown on the state of mental health services in the private sector in India. Indian J Psychiatry. 2020 Sep-Oct;62(5):488-93. doi: 10.4103/psychiatry.IndianJPsychiatry_568_20, PMID 33678828.

4. Grover S, Vaishnav M, Tripathi A, Rao TSS, Avasthi A, Dalal PK, et al. Sexual functioning during the lockdown period in India: an online survey. Indian J Psychiatry. 2021;63(2):134-41. doi: 10.4103/psychiatry. IndianJPsychiatry_860_20, PMID 34194056, P. K, et al.

5. Grover S, Sahoo S, Mehra A, Avasthi A, Tripathi A, Subramanyan A, et al. Psychological impact of Covid-19 lockdown: An online survey from India. Indian J Psychiatry. 2020 Jul-Aug;62(4):354-362.

6. Kumar K, Mehra A, Sahoo S, Nehra R, Grover S. The psychological impact of Covid-19 pandemic and lockdown on the migrant workers: A cross-sectional survey. Asian J Psychiatr. 2020;53:102252. doi: 10.1016/j.ajp.2020.102252.

7. Mazza M, Marano G, Lai C, Janiri L, Sani G. Danger in danger: interpersonal violence during Covid-19 quarantine. Psychiatry Res. 2020;289. doi: 10.1016/j. psychres.2020.113046, PMID 113046.

8. Suresh R, James J, R S J B. Migrant Workers at Crossroads-The Covid-19 Pandemic and the Migrant Experience in India. Soc Work Public Health. 2020 Sep 1;35(7):633-43. doi: 10.1080/19371918.2020.1808552, PMID 32970543. Rogers JP, Chesney E, Oliver D, Pollak T. A, McGuire, P, Fusar-Poli, P, Zandi, M. Lewis, G, and David, A. S. Psychiatric and neuropsychiatric presentations associated with severe coronavirus infections: A systematic review and meta-analysis with comparison to the Covid-19 pandemic. Lancet Psychiatry. $2020 \mathrm{Jul} ; 7(7): 611-627$

9. Taquet M, Geddes JR, Husain M, Luciano S, Harrison PJ. 6-month neurological and psychiatric outcomes in 236379 survivors of Covid-19: A retrospective cohort study using electronic health records. Lancet Psychiatry. 2021;8(5):416-27. doi: 10.1016/S2215-0366(21)00084-5, PMID 33836148

10. Maalouf FT, Mdawar B, Meho LI, AkI EA. Mental health research in response to the Covid-19, Ebola, and H1N1 outbreaks: A comparative bibliometric analysis. J Psychiatr Res. Jan 2021;132:198-206. doi: 10.1016/j.jpsychires.2020.10.018 PMID 33131830.

11. Gul S, Rehman SU, Ashiq M, Khattak A. Mapping the scientific literature on Covid-19 and mental health. Psychiatr Danub. 2020 Autumn-Winter: 32(3-4):463-71. doi: 10.24869/psyd.2020.463, PMID 33370754.

12. Dubey MN, Dadhe PP. Mapping the scholarship on mental health during Covid-19 pandemic: A scientometric view Library Philosophy and PracticeElectronic [journal]. https:

13. Grover S, Gupta B. M, Mamdapur, G, Mehra, A and Sahoo, S. The impact of Covid-19 on mental health: A global analysis of publications. Submitted for publication.

14. Saxena S, Paraje G, Sharan P, Karam G, Sadana R. The 10/90 divide in mental health research: trends over a 10-year period. Br J Psychiatry. 2006 Jan;188:81-2 doi: 10.1192/bjp.bp.105.011221, PMID 16388075.

15. Grover S, Gupta BM, Dhawan SM. Schizophrenia research in India: A scientometric assessmentof India's publications during 1990-2019. Asian J Psychiatr. 2021 Feb;56. doi: 10.1016/j.ajp.2020.102521, PMID 102521.

16. Grover S, Gupta BM, Dhawan SM. Research on bipolar disorder from India: 
A bibliometric analysis of papers published during 2000-19. Asian J Psychiatr. 2021 Jan;55. doi: 10.1016/j.ajp.2020.102532, PMID 102532.

17. Luo M, Guo L, Yu M, Jiang W, Wang $H$. The psychological and mental impact of coronavirus disease 2019 (Covid-19) on medical staff and general public - A systematic review and meta-analysis. Psychiatry Res. 2020 Sep;291:113190. doi: 10.1016/j.psychres.2020.113190.

18. Tandon R. The Covid-19 pandemic, personal reflections on editorial responsibility. Asian J Psychiatr. 2020;50:102100. doi: 10.1016/j.ajp.2020.102100.

Article History: Received: 12-08-2021; Revised: 19-09-2021; Accepted: 20-10-2021.

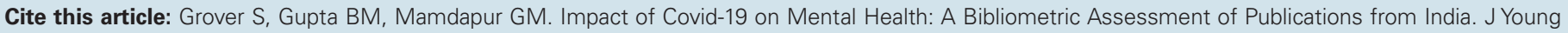
Pharm. 2021;13(3) Suppl:s66-s71. 